\title{
PRESENT STATUS OF PERIPHERAL NEURECTOMY FOR PAIN IN OBLITERATIVE ARTERIAL DISEASE*
}

\author{
ALEXANDER BLAIN III, M.D., NESTER A. FLORES, M.D. AND \\ FRANCIS GERBASI, M.D.
}

\author{
From the Departments of Surgery, Alexander Blain Hospital, Detroit, and the University of \\ Michigan, Ann Arbor, Michigan
}

It is not generally known that section of peripheral nerves is available as a therapeutic measure in a number of patients suffering from intractable pain resulting from obliterative disease of the arteries. The term "peripheral neurectomy" usually is connected with the therapy of intractable pain associated with the supra-orbital, glossopharyngeal, greater and lesser occipitals and other nerves in the head.

The purpose of this communication is to point out that in the treatment of occlusive arterial disease of the extremities, particularly the lower extremities, peripheral neurectomy occupies a significant, although limited place, when intractable pain occurs. Recourse to the surgical literature or to textbooks of surgery will be of little aid to the physician who desires to learn the uses and limitations of this surgical measure, because so little has been written about it.

By "intractable" pain is meant the severe rest pain which is frequently seen in cases of arteriosclerosis obliterans and thromboangiitis obliterans, particularly when there is localized ulceration or gangrene of the toes or distal portions of the foot. In patients having thromboangitis obliterans, the blood supply to the remainder of the limb may well be adequate; but in patients with arteriosclerosis obliterans, intractable pain is often indicative of complete arterial insufficiency. In either case, there are far more limbs lost each year by amputation than need be. Frequently, concentrated efforts with conservative measures can salvage limbs which would otherwise be lost. These measures include cessation of smoking, reflex vasodilatation, vasodilator drugs, Buerger-Allen exercises, antibiotics when indicated, control of organic diseases such as diabetes mellitus, and therapy for functional diseases when present, together with the increasing use of lumbar sympathectomy (1) and, in some cases, peripheral neurectomy $(2,3,4)$.

\section{HISTORICAL CONSIDERATIONS}

In 1922, Silbert (5) reported the injection of alcohol into the posterior tibial nerve for relief of pain in 3 patients with thromboangiitis obliterans. The alcohol was injected under direct vision through an incision at the level of the internal malleolus, through which the nerve was exposed. Eleven years later, Laskey and Silbert (6) reported sectioning (with immediate resuturing) of the peripheral nerves in 13 patients suffering from pain due to thromboangiitis obliterans. They described, in a few patients, a residual dull aching and occasional sharp, shooting

Received for publication December 11, 1952.

* Presented at the Sixth Annual Meeting of the American Society for the Study of Arteriosclerosis, Chicago, Ill., Nov. 9-10, 1952. 
pain, thought to be due to nerve-end irritation but in general observed that the pain was markedly relieved. In the meantime, Smithwick and White (2) in 1930 described a technique for injecting the peripheral nerves of the lower extremity at higher levels in the leg, in an attempt to promote better wound healing. They were able, without sacrificing important motor fibers to the muscles of the lower leg, to relieve pain completely with good wound healing at these higher levels of incision, when the proper combination of nerves to inject was selected. Of 11 cases, pain was relieved in 9 . They reported that 6 of 11 extremities had been sàved from major amputation by relieving pain.

Allen (3) in 1932 reported the injection or crushing of the peripheral nerves in 29 patients with thromboangiitis obliterans, with relief of pain in all cases. He stated that major amputations had been reduced by 50 per cent in this series.

In 1935, Smithwick and White (4) recommended crushing the peripheral nerves, as easier and more satisfactory than alcohol injections. In 45 patients treated by either alcohol injection or crushing, 28 had thromboangiitis obliterans and 17 suffered from arteriosclerosis obliterans. Among the 17 patients with arteriosclerosis obliterans, 10 major amputations (58.8 per cent) were performed, whereas in the 28 patients with thromboangiitis obliterans, 12 major amputations were later performed on 34 involved extremities (only 35.3 per cent). They concluded that major amputation was not always necessary for the relief of intractable pain. They stated that although nerve block was effective in relieving pain in advanced obliterative disease, results were better in thromboangiitis obliterans than in arteriosclerosis obliterans. It was their impression that peripheral nerve block or crushing doubled the number of successful minor amputations of ulcerated or gangrenous toes and that it reduced subsequent major amputations to one third of the number necessary previously (7).

More recently Orebaugh and Gustafson (8) have confirmed the discrepancy between the results of peripheral neurectomy in patients with arteriosclerosis obliterans and in those with thromboangiitis obliterans, the latter having much more dramatic pain relief and a lower incidence of major amputation. They reported that of 45 patients with arteriosclerosis obliterans treated at the University of Michigan Hospital by peripheral neurectomy, only a small percentage obtained pain relief of any appreciable duration. Of those patients whose follow-up study was adequate, only 8 (17.2 per cent) were partially or completely relieved of pain and still avoided major amputation. Eleven of 12 diabetic patients in this group required supracondylar amputation. On the other hand, all 16 patients with thromboangiitis obliterans in their study were relieved partially or completely by peripheral neurectomy. Ten of these patients (62.4 per cent) avoided major amputation.

Rarely, peripheral neurectomy is indicated in the relief of pain in ulcerated or gangrenous digits in the upper extremities. Sensory nerves to the dorsum of the hand may be sectioned or crushed at the wrist, and digital branches of the ulnar and median nerves may be sectioned at points distal to their important motor branches, through small incisions in the palm. We have observed good pain relief in 2 cases of far-advanced Raynaud's disease, after failure of dorsal sympathectomy (complete, as determined by sweating tests) to abolish pain 
in established gangrene of digits (9). The senior author has had occasion to perform peripheral neurectomy only once in the upper extremity for the relief of intractable pain associated with ulcerated digits in thromboangiitis obliterans. In this case, section of the digital branches of the ulnar nerve of the left hand completely relieved the pain and appeared to facilitate healing of the ulcers.

In a brief communication in 1952, Blain and Gerbasi, basing their conclusions on observations of the Ann Arbor series as well as of 10 cases in Detroit (4 with thromboangiitis obliterans and 6 with arteriosclerosis obliterans), suggested that peripheral neurectomy be abandoned in patients having intractable pain resulting from arteriosclerosis obliterans (10). Although we still maintain that in a practical way this may be true, we would emphasize that there is nothing to lose by a trial of this operation in those cases in which the only alternative is a major amputation.

\section{ANATOMY AND PIYYSIOLOGY}

The sensory nerve supply to the foot is derived from the lumbosacral plexus, chiefly via terminal branches of the sciatic nerve $\left(L_{4,5}\right.$, and $\left.S_{1,2,3}\right)$, but also receives a branch from the femoral nerve $\left(\mathrm{L}_{2,3}, 4\right)$. Nerves from the sciatic include the tibial (medial plantar, lateral plantar, vascular branches and the sural nerve) and the peromeal (superficial peroneal and deep peroneal or anterior tibial). From the femoral nerve is derived the saphenous nerve.

Of these nerves the saphenous and sural are purely sensory, and the motor branches of the superficial and deep peroneal nerves are given off high in the leg so that they are usually sensory in the distal third of the leg and foot. The tibial nerve is a mixed one and in addition to its sensory fibers, supplies motor fibers to the intrinsic muscles of the foot. There is practically no disability, however, resulting from the paralysis of these muscles when this nerve is sectioned.

Technique. We prefer to section the peripheral nerves at the level of the junction of the middle and lower thirds of the leg in most cases, as healing is more assured than in more distal incisions, particularly in the presence of arteriosclerosis obliterans with absent popliteal artery pulsations. The operative approach may be summarized as follows:

Tibial nerve. A short vertical incision is made four to five inches above the medial malleolus (distal to the last muscular branch--that to the flexor hallucis longus); this incision should be about two centimeters posterior to the posterior margin of the tibia. After incising the deep fascia, the cleavage plane between the soleus and the flexor digitorum longus is developed. The artery and vein are exposed and the nerve is usually posterior and lateral to them.

Superficial and deep peroneal nerves. A similar incision is made four to five inches above the lateral malleolus and through this the superficial peroneal nerve can be found in the subcutaneous tissue. Section must be above the point of bifurcation of this nerve into medial and lateral cutaneous branches. The deep peroneal nerve is exposed by incising the deep fascia and developing the plane between the tibialis anticus and extensor hallucis longus muscles. The nerve is usually small, and superficial to the anterior tibial artery and vein.

Sural nerve. A vertical incision is made in the midline of the posterior aspect 
of the lower leg, four to seven inches above the ankle. The nerve is found in the subcutaneous tissue in close proximity to the lesser saphenous vein.

Saphenous nerve. Through the same incision made for exposure of the tibial nerve, the saphenous nerve is exposed in the subcutaneous tissue accompanying the greater saphenous vein.

In our opinion, a segment of about 2 centimeters of each nerve should be resected, as this is more certain than crushing. General or spinal anesthesia is preferred to local infiltration anesthesia.

There are several physiologic factors which should be mentioned. Although important, these are not yet well understood. The first is the mechanism of pain in obliterative arterial disease. In the type of pain under discussion, "intractable pain," we are not concerned with intermittent claudication or with so-called ischemic neuritis, but rather with that severe type of rest pain, either pretrophic or associated with ulceration and gangrene, which does not respond to any form of conservative therapy. The agonized patient frequently demands amputation and this is true whether or not sympathectomy has been performed. Rest pain is generally believed to be due to ischemia and the painful stimuli arise not only in ulcerated and dying skin and soft tissues but also, as demonstrated by Leriche (11), in blood vessels.

Section of peripheral nerves interrupts the passage of painful impulses over afferent sensory nerve fibers from involved areas and also produces a transient vasodilatation which relieves ischemia. In patients requiring peripheral neurectomy even after lumbar (or rarely dorsal) sympathectomy, it is obvious that relief of ischemia is not enough or that it is impossible. Peripheral neurectomy does, however, interrupt afferent fibers which were not interrupted by the sympathectomy. Furthermore, the vasodilatation following neurectomy is transient and although Smithwick and White believed that relief of ischemia was one of the chief effects of neurectomy, it was subsequently demonstrated by Atlas (12) and by Schulenberg (13) that the vasodilatation following peripheral neurectomy is transient and is followed by permanent vasoconstriction in about three weeks.

\section{THERAPEUTIC INDICATIONS}

We are in agreement with Orebaugh and Gustafson that peripheral neurectomy should not be carried out until sympathectomy has been followed by failure to relieve pain. Only after sympathectomy has been performed is it known in these cases that pain persists in spite of the best vasodilatation which can be produced under the circumstances. It is important to point out that although pain arising in visceral arteries is transmitted over sensory axons in the splanchnic and sympathetic nerves, painful stimuli from peripheral arteries traverse the spinal nerves, as shown by Moore and Singleton (14). Sympathectomy then acts by relieving vasospasm but peripheral neurectomy acts by interruption of afferent sensory fibers.

Finally, it should be emphasized that sympathectomy should be performed first in patients with symptomatic arteriosclerosis obliterans unrelieved by conservative measures (Blain and Campbell (1)). The same rule applies in thrombo- 
angiitis obliterans (Campbell, Harris and Coller (15)). Frequently relief will be obtained and limbs will be salvaged. In the group of cases in which intractable pain, as defined in this article, is unrelieved by sympathectomy, peripheral neurectomy should be considered before amputation is decided upon. As mentioned, results, will be more striking in thromboangiitis obliterans than in arteriosclerosis obliterans, but there is nothing to lose by a trial of the operation in the latter case, when amputation is the only alternative.

\section{CONCLUSIONS}

1. Attention is drawn to the fact that peripheral neurectomy is available as an established but not widely known surgical procedure for the relief of intractable pain resulting from obliterative arterial disease.

2. The development, rationale, results, anatomic and physiologic considerations, and technique of peripheral neurectomy are briefly reviewed.

3. This operation should be considered only after good conservative therapy and the production of maximal vasodilatation by sympathectomy to relieve ischemia, has failed to relieve pain.

4. Peripheral neurectomy relieves pretrophic and trophic rest pain or "intractable pain" in thromboangiitis obliterans and arteriosclerosis obliterans by interruption of afferent sensory fibers which carry painful stimuli via spinal nerves.

5. Results of this operation in relieving pain and saving limbs are superior in thromboangiitis obliterans and disappointing in arteriosclerosis obliterans. If, however, even after sympathectomy, pain is so severe in arteriosclerosis obliterans that a major amputation is the only alternative, there is nothing to lose by a trial of peripheral neurectomy.

\section{REFERENCES}

1. Blain, A., III, and Campbell, K. N.: Lumbar sympathectomy for arteriosclerosis obliterans. Rationale and results, Surgery 25: 950, 1949.

2. Smiтhwick, R. H., AND White, J. C.: Elimination of pain in obliterative vascular disease of the lower extremities, Surg., Gynec. \& Obst. 51: 394, 1930.

3. Allen, A. W. : Results obtained in the treatment of Raynaud's disease by sympathetic neurectomy and in thromboangiitis obliterans by desensitization of peripheral sensory nerves, Ann. Surg. 96: 867, 1932.

4. Smithwick, R. H., and White, J. C.: Peripheral nerve block in obliterative vascular disease of the lower extremity, Surg., Gynec. \& Obst. 60: 1106, 1935.

5. Sicbert, S.: A new method for the treatment of thromboangiitis obliterans, J.A.M.A 79: 1765,1922 .

6. Laskex, N. F., AND SilberT, S. : Thromboangiitis obliterans; relief of pain by peripheral nerve section, Ann. Surg. 98: 55, 1933.

7. White, J. C., and Smithwick, R. H.: The Autonomic Nervous System, ed. 2. New York, The MacMillan Co., 1941.

8. Orebaugh, J., and Gustafson, J. R.: Peripheral neurectomy: its use in relieving the pain of obliterative peripheral vascular diseases, Univ. Michigan Med. Bull. 16: 197, 1950.

9. Blain, A., III; Coller, F. A., and Carver, G. B.: Raynaud's disease. A study of criteria for prognosis, Surgery 29: 387, 1951. 
10. Biaix, A., III, and Gerbasi, F. S.: Peripheral neurectomy for pain in obliterative arterial disease, Circulation 6: 471, 1952.

11. Leriche, Rene: Surgery of Pain. Baltimore, The Williams \& Wilkins Co., 1939.

12. Atras, L. N.: Further observations on the etiology of vasomotor disturbances following peripheral nerve section, Surgery 10:318, 1941.

13. Schulenburg, C. A. R.: Vasomotor changes in peripheral nerve injuries, \$urgery 25: $191,1949$.

14. Moore, R. M., and Singleton, A. D., JR.: Studies on the pain-sensibility of arteries. II. Peripheral paths of afferent neurones from the arteries of the extremities and of the abdominal viscera, Am. J. Physiol. 104: 267, 1933.

15. Campbell, K. N.; Harris, B. M., and Coller, F. A.: A follow up study of patients with thromboangiitis obliterans (Buerger's disease), Surgery 26: 1003, 1949. 